\title{
AN ACCELERATION PROCEDURE OF REGULA FALSI METHOD
}

\author{
M. A. HERNÁNDEZ AND M. A. SALANOVA
}

\begin{abstract}
From the Regula Falsi method a family of iterative processes is defined. A family of accelerations is obtained, by means of a geometric procedure. From this, a family of new iterative processes with, at least, quadratic convergence is derived. A study of their convergence and optimization is done in $\mathbb{R}$ and in the complex plane. All of them are better than Newton method.
\end{abstract}

\section{Introduction}

Regula Falsi method for solving a nonlinear equation

$$
f(x)=0
$$

is well know [4]. It is given by the expression

$$
x_{n}=x_{n-1}-f\left(x_{n-1}\right) \frac{\lambda-x_{n-1}}{f(\lambda)-f\left(x_{n-1}\right)} .
$$

If $f \in C^{(2)}([a, b])$, satisfying Fourier's conditions, i.e. $f(a) f(b)<0, f^{\prime} \neq 0$, the sign of $f^{\prime \prime}$ is constant in $[a, b]$, and $x_{0}, \lambda \in[a, b]$ such that $f(\lambda) f^{\prime \prime}(\lambda)>0$ and $f\left(x_{0}\right) f^{\prime \prime}\left(x_{0}\right)<0$, then the sequence $\left\{x_{n}\right\}$, given by (1.2) converges to the unique root $s$ of (1.1) in $[a, b]$. This procedure has linear convergence.

In this paper, first we study the family of iterative processes, generalization of Regula Falsi method, given by

$$
x_{n}=F_{\mu}\left(x_{n-1}\right)=x_{n-1}-f\left(x_{n-1}\right) \frac{\lambda-x_{n-1}}{\mu-f\left(x_{n-1}\right)}
$$

where $\lambda \in[a, b]$ and $\mu$ is a variable real parameter. Let us note that when $\mu=f(\lambda)$, we obtain Regula Falsi method.

We give theorems on convergence and optimization for this family and prove that all of these processes have linear convergence. As a consequence of these results we

Received January 5, 1996.

1991 Mathematics Subject Classification. 65H05, 26A51.

Key words and phrases Nonlinear equation, Regula Falsi method, Kantorovich assumptions, iterative processes, error bounds.

Supported in part by a grant of the University of La Rioja. 
derive a new theorem of convergence for Regula Falsi method. Besides, by means of an optimization study, we get some new iterative processes with linear convergence better than Regula Falsi method and with the same efficiency index [4].

Then, a new family of accelerations with quadratic convergence is derived using a geometric procedure to accelerate (1.3). As a particular case an acceleration of the Regula Falsi method is given.

As these accelerations are in the form $y_{n}=G_{\mu}\left(x_{n-1}\right)$, they define a new family of iterative processes $t_{n}=G_{\mu}\left(t_{n-1}\right)$. A Theorem of convergence and optimization for them is given. All of them have, at least, quadratic order of convergence and are better than Newton's method, as we show with an example.

Finally we extend this family to the Complex plane and derive some convergence and uniqueness results.

\section{A Family of New Iterative Processes with Linear Convergence}

In this Section we find the values of $\mu$ for which the sequence $\left\{x_{n}\right\}$, given by (1.3), converges to the root of (1.1).

From now, let $f:[a, b] \subseteq \mathbb{R} \rightarrow \mathbb{R}$ be a function $f \in C^{(2)}([a, b])$, such that $f^{\prime}(x)>0$, $f^{\prime \prime}(x) \geq 0$, for $x \in[a, b]$ and $f(a) f(b)<0$. Let $\lambda \in[a, b]$ such that $f(\lambda)>0$. Notice that if $f$ verifies Fourier's conditions and the above hypothesis are not satisfied we can replace $f(x)$ by $f(-x),-f(x)$ or $-f(-x)$.

Theorem 2.1. Let $f$ and $\lambda$ like in the above conditions, $x_{0} \in(a, b], f\left(x_{0}\right)>0$ and $\lambda>x_{0}$. Fixed $\mu$ such that $\mu \geq f\left(x_{0}\right)+f^{\prime}\left(x_{0}\right)\left(\lambda-x_{0}\right)$ then, the sequence $\left\{x_{n}\right\}$ given by (1.3) is decreasing to $s$.

Proof. As $f\left(x_{0}\right)>0$, we obtain that $F_{\mu}^{\prime}(x)>0$ in $\left(s, x_{0}\right]$ since that

$$
F_{\mu}^{\prime}(x)=\frac{\mu}{\mu-f(x)}\left[1-f^{\prime}(x) \frac{\lambda-x}{\mu-f(x)}\right] .
$$

On the other hand, as

$$
f\left(x_{0}\right)=f(x)+f^{\prime}(x)\left(x_{0}-x\right)+\frac{f^{\prime \prime}(\theta)}{2}\left(x_{0}-x\right)^{2},
$$

we have

$$
\mu-f(x) \geq f^{\prime}(x)(\lambda-x)+\left(x_{0}-x\right)^{2} \frac{f^{\prime \prime}(\theta)}{2}>f^{\prime}(x)(\lambda-x)
$$

with $\theta \in\left(x, x_{0}\right)$.

Therefore applying induction and the Mean Value Theorem, we have $x_{n} \geq s$ for $n \in \mathbb{N}$. Besides, as

$$
x_{n}-x_{n-1}=-f\left(x_{n-1}\right) \frac{\lambda-x_{n-1}}{\mu-f\left(x_{n-1}\right)} \leq 0
$$

for $n \in \mathbb{N}$, the result holds. 
Corollary 2.2. In the previous conditions, let

$$
\mu_{1}>\mu_{2} \geq f\left(x_{0}\right)+f^{\prime}\left(x_{0}\right)\left(\lambda-x_{0}\right)
$$

$\left\{x_{n, 1}\right\}$ and $\left\{x_{n, 2}\right\}$ the sequences given by (1.3) for $\mu=\mu_{1}$ and $\mu=\mu_{2}$ respectively, with $x_{0}=x_{0,1}=x_{0,2}<\lambda$ and $f\left(x_{0}\right)>0$. Then, the sequence $\left\{x_{n, 2}\right\}$ converges to $s$ faster than $\left\{x_{n, 1}\right\}$.

Proof. Taking into account that $f\left(x_{0}\right)>0$ we have

$$
x_{1,2}-x_{1,1}=f\left(x_{0}\right)\left(\lambda-x_{0}\right)\left[\frac{1}{\mu_{1}-f\left(x_{0}\right)}-\frac{1}{\mu_{2}-f\left(x_{0}\right)}\right]<0 .
$$

Then, as $F_{\mu_{2}}$ is an increasing function in $(s, b]$ we derive by induction $x_{n, 2}<x_{n, 1}$ for $n \in \mathbb{N}$.

Observe that for $\mu=f(\lambda)$ we obtain Regula Falsi method. Besides the iterative processes of the family (1.3) have linear convergence and they converge to the root of (1.1) faster than Regula Falsi method for $\mu \in\left[f\left(x_{0}\right)+f^{\prime}\left(x_{0}\right)\left(\lambda-x_{0}\right), f(\lambda)\right)$. Notice that

$$
f(\lambda)=f\left(x_{0}\right)+f^{\prime}\left(x_{0}\right)\left(\lambda-x_{0}\right)+\frac{1}{2} f^{\prime \prime}(\theta)\left(\lambda-x_{0}\right)^{2}
$$

with $\theta \in\left(x_{0}, \lambda\right)$.

Now, we are going to apply a geometric procedure of acceleration to the family given by (1.3). In the same conditions.of Theorem 2.1 the sequence $\left\{x_{n}\right\}$ is decreasing to $s$. Now we get an acceleration $\left\{y_{n}\right\}$ from $\left\{x_{n}\right\}$. Fixed $\mu \geq f\left(x_{0}\right)+f^{\prime}\left(x_{0}\right)\left(\lambda-x_{0}\right)$, consider

$$
y_{n}=x_{n-1}-f\left(x_{n-1}\right) \frac{\lambda-x_{n-1}}{\mu_{n-1}-f\left(x_{n-1}\right)}
$$

where the values of $\mu_{n-1}$ are obtained in the following way: $\mu_{0}=\mu$ and for each $i \in \mathbb{N}$, $\mu_{i}$ is given by the point $P=\left(\lambda, \mu_{i}\right) \in \mathbb{R}^{2}$, intersection of the straight lines,

$$
x=\lambda
$$

and

$$
y=f\left(x_{i}\right)+f\left(x_{i}\right) \frac{x-x_{i}}{x_{i}-s}
$$

(see Figure 1). Thus

$$
\mu_{i}=f\left(x_{i}\right)+f\left(x_{i}\right) \frac{\left(\lambda-x_{i}\right)}{x_{i}-s} .
$$

The problem is that $s$ is unknown. If we substitute in $(2.2), x_{i}-s$ for its equivalent expression

$$
x_{i}-s \approx \frac{f^{\prime}\left(x_{i}\right)\left(\lambda-x_{i}\right)}{x_{i-1}-x_{i}}
$$




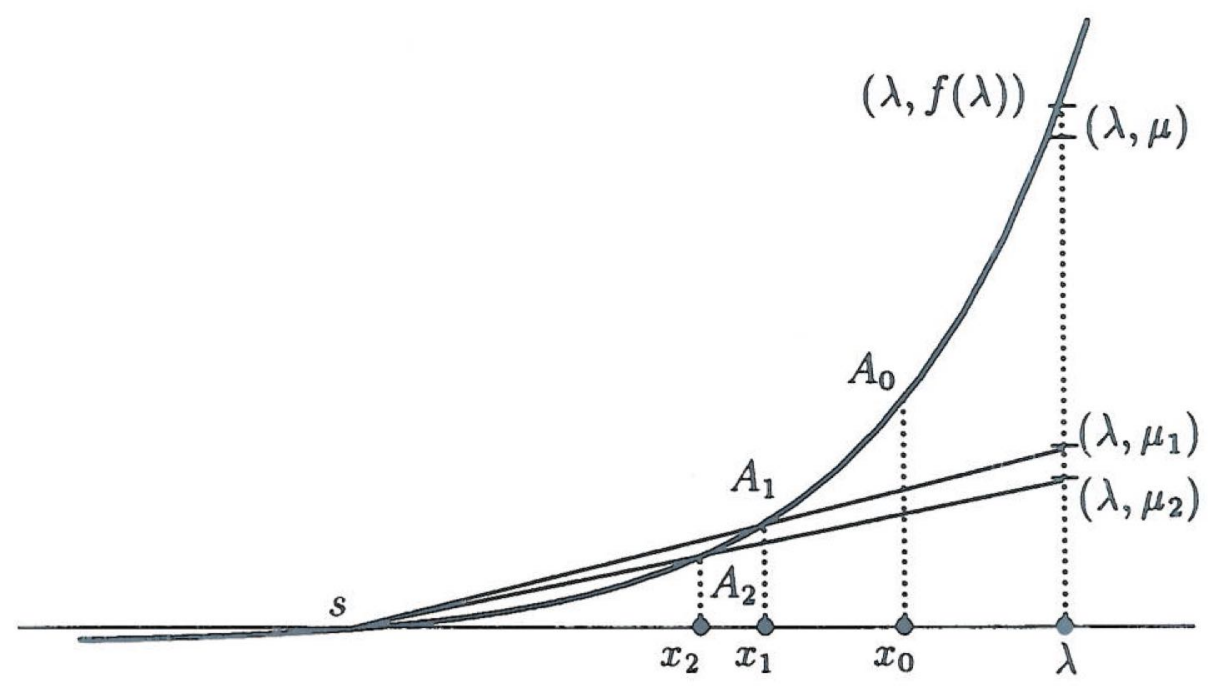

Figure 1 .

we obtain

$$
\mu_{i}=f\left(x_{i}\right)+\frac{f^{\prime}\left(x_{i}\right)\left(\mu-f\left(x_{i}\right)\right)\left(\lambda-x_{i}\right)}{\mu} .
$$

From (2.1) we derive an acceleration for $\left\{x_{n}\right\}$ given by the expression

$$
y_{n}=G_{\mu}\left(x_{n-1}\right)=x_{n-1}-\frac{f\left(x_{n-1}\right) \mu}{f^{\prime}\left(x_{n-1}\right)\left(\mu-f\left(x_{n-1}\right)\right)} .
$$

Theorem 2.3. The sequence $\left\{y_{n}\right\}$, given by (2.3), is an acceleration of $\left\{x_{n}\right\}$ with, at least, quadratic convergence.

Proof. We can prove immediately that $\lim _{n \rightarrow \infty} y_{n}=\lim _{n \rightarrow \infty} x_{n}=s$ and since $F_{\mu}^{\prime} \neq 0$ and $G_{\mu}^{\prime}(s)=0$ we get

$$
\lim _{n \rightarrow \infty} \frac{\left|y_{n}-s\right|}{\left|x_{n}-s\right|}=0
$$

\section{A Family of New Iterative Processes of Second Order}

Let $f$ be as in the last Section. From the accelerations (2.3) we can define a family of new iterative processes as follows

$$
t_{n, \mu}=G_{\mu}\left(t_{n-1, \mu}\right)=t_{n-1, \mu}-\frac{f\left(t_{n-1, \mu}\right) \mu}{f^{\prime}\left(t_{n-1, \mu}\right)\left(\mu-f\left(t_{n-1, \mu}\right)\right)} .
$$

where $\mu>0$ is a variable real parameter. In the next result we give conditions about the convergence of (3.1). For this, we consider the degree of logarithmic convexity of $f$, a punctual measure of the convexity, introduced in $[1]$. Let $f:[a, b] \subseteq \mathbb{R} \rightarrow \mathbb{R}$ be a convex, 
twice differentiable function on an interval $[a, b]$ and $t_{0} \in[a, b]$ such that $f^{\prime}\left(t_{0}\right) \neq 0$. The degree of logarithmic convexity of $f$ at $t_{0}$ is

$$
L_{f}\left(t_{0}\right)=\frac{f\left(t_{0}\right) f^{\prime \prime}\left(t_{0}\right)}{f^{\prime}\left(t_{0}\right)^{2}} .
$$

Denote

$$
U[f](c)=\frac{f^{\prime \prime}(c)}{f^{\prime}(c)^{2}}
$$

the degree of logarithmic convexity of the function $T[f](t)=f(t)-f(c)+1$ in the point c. Notice that $L_{f}(c)=f(c) U[f](c)$. Denote $m=\min \{U[f](t) \mid t \in[a, b]\}$, and consider $t_{0} \in[a, b]$ with $f\left(t_{0}\right)>0$.

Theorem 3.1. If $\mu \geq f\left(t_{0}\right)+\frac{2}{m}$, the iterative process given by (3.1) for $t_{0, \mu}=t_{0}$ is decreasing to $s$.

Proof. We derive from the hypothesis that $G_{\mu}$ is an increasing function in $(s, b]$ since that

$$
G_{\mu}^{\prime}(t)=\frac{\mu^{2} L_{f}(t)-2 \mu f(t)-\mu L_{f}(t) f(t)+f(t)^{2}}{[\mu-f(t)]^{2}} .
$$

The induction procedure assure that the sequence $\left\{t_{n, \mu}\right\}$ is bounded by $s$.

On the other hand, we have

$$
t_{0}-t_{1, \mu}=\frac{\mu f\left(t_{0}\right)}{f^{\prime}\left(t_{0}\right)\left(\mu-f\left(t_{0}\right)\right)} \geq 0
$$

since $\mu-f\left(t_{0}\right) \geq \frac{2}{m}>0$. In a similar way we obtain $t_{n, \mu}-t_{n-1, \mu} \geq 0$. Thus, $\left\{t_{n, \mu}\right\}$ converges to $u \in[a, b]$. Making $n \rightarrow \infty$ in (3.1), we have $f(u)=0$. As $f$ has unique root in $[a, b]$, we conclude $u=s$.

Now, we give an optimization result.

Corollary 3.2. Under the assumptions of the last theorem, let $\mu_{1}$ and $\mu_{2}$ such that, $f\left(t_{0}\right)+\frac{2}{m} \leq \mu_{1}<\mu_{2}, t_{0}=t_{0,1}=t_{0,2}$ and $\left\{t_{n, 1}\right\},\left\{t_{n, 2}\right\}$ the respective sequences obtained by (3.1) for these values of $\mu$. Then, $t_{n, 1}<t_{n, 2}$ for all $n \in \mathbb{N}$.

Proof. As $G_{\mu}$ is an increasing function, the proof is analogous to the one of Corollary 2.2 .

As a consequence of the previous results, the best sequence $\left\{t_{n, \mu}\right\}$ in (3.1) is obtained for $\mu=f\left(t_{0}\right)+\frac{2}{m}$. Furthermore, as $G_{\mu}(s)=s$ and $G_{\mu}^{\prime}(s)=0$ the processes (3.1) have, at least, quadratic convergence.

Finally, we compare the iterative processes (3.1) with Newton method by means the asymptotic error constants [2]. We denote

$$
C_{1}=\left|\frac{f^{\prime \prime}(s)}{2 f^{\prime}(s)}\right|
$$


the Newton asymptotic error constant and

$$
C_{2}=\left|\frac{G_{\mu}^{\prime}(s)}{2}\right|=\left|\frac{f^{\prime \prime}(s)}{2 f^{\prime}(s)}-\frac{f^{\prime}(s)}{\mu}\right|,
$$

the asymptotic error constant for the iterative processes (3.1). Then, derive $C_{2}<C_{1}$ since $\mu \geq \frac{2}{m}$. Thus, all the iterative processes (3.1) converge to the root of (1.1) faster than Newton method. Notice that we obtain Newton method when $\mu \rightarrow \infty$.

Now we are going to realize a practical comparative study between Newton's method and the family given by (3.1). For this, we consider the nonlinear equation

$$
x^{p}-r=0, \text { for } p>1, r, x \in \mathbb{R}, r>0 \text { and } x \geq 0 .
$$

Table 1. Error bounds

\begin{tabular}{|c|c|c|}
\hline$n$ & $t^{*}-t_{n}$ & $t^{*}-x_{n}$ \\
\hline 0 & 0.1448177147 & 0.1448177147 \\
1 & 0.0895581894 & 0.0651290799 \\
2 & 0.0434378457 & 0.0234392078 \\
3 & 0.0129149572 & 0.0039386555 \\
4 & 0.0013624215 & 0.0001264288 \\
5 & 0.0000162942 & $1.336710^{-7}$ \\
6 & $2.3507210^{-9}$ & $1.4954710^{-13}$ \\
\hline
\end{tabular}

Then, for $f(x)=x^{p}-r$, and $x>0$ it follows that $U[f](x)=\frac{p-1}{p x^{p}}$ is a decreasing function.

Therefore, if we consider, for example, $p=25, r=1995$ and apply the Theorem 3.1 for $[1,1.5], x_{0}=1.5$ and

$$
\mu=f\left(x_{0}\right)+2 / m=f(1.5)+2 / U[f](1.5)=75862.8,
$$

thus from (3.1) we have the sequence $\left\{x_{n}\right\}$.

On the other hand, let $\left\{t_{n}\right\}$ the sequence obtained by Newton's method to the same equation, with $t_{0}=x_{0}=1.5$. Then we have the results of the Table 1 . It shows the error between the exact value and the respective sequences. The calculations were made with 20 decimal places and

$$
t^{*}=1.355182285290081946 \text {. }
$$

This shows us the increment in the velocity of convergence taking into account the simplicity of the equation considered.

\section{Convergence Under $\mathbb{K a n t o r o v i c h ~ T y p e ~ C o n d i t i o n s ~ i n ~ t h e ~ C o m p l e x ~ P l a n e ~}$}

Let $h: \Omega \rightarrow \mathbb{C}$ be an holomorphic function in an open convex domain $\Omega \subseteq \mathbb{C}$. For solving the equation

$$
h(z)=0
$$


we can use the generalization of (3.1) given by

$$
z_{n, \mu}=z_{n-1, \mu}-\frac{h\left(z_{n-1, \mu}\right) \mu}{h^{\prime}\left(z_{n-1, \mu}\right)\left(\mu-h\left(z_{n-1, \mu}\right)\right)}
$$

where $z_{0, \mu} \in \Omega$ and $\mu>0$.

We assume throughout this Sections that

[I] $\left|h\left(z_{0}\right)\right| \leq a$, and $\left|\frac{1}{h^{\prime}\left(z_{0, \mu}\right)}\right| \leq b$.

[III] There exist $k>0$ such that the equation

$$
p(t)=\frac{k}{2} t^{2}-\frac{t}{b}+a
$$

has two positive roots $t^{*}$ and $t^{* *}\left(t^{*} \leq t^{* *}\right)$. Equivalently, $2 k a b^{2} \leq 1$, where the equality holds if and only if $t^{*}=t^{* *}$.

Let us define the scalar sequence $\left\{t_{n, \mu}\right\}$ by

$$
t_{0, \mu}=0, t_{n, \mu}=P_{\mu}\left(t_{n-1, \mu}\right)=t_{n-1, \mu}-\frac{p\left(t_{n-1, \mu}\right) \mu}{p^{\prime}\left(t_{n-1, \mu}\right)\left(\mu-p\left(t_{n-1, \mu}\right)\right)}
$$

and for each $\mu \geq a+\frac{2}{k}$, where $p(t)$ is the polynomial defined by (4.3). Notice that $k=\min \left\{U[p](t) \mid t \in\left[t_{0, \mu}, t^{*}\right]\right\}$.

Under assumptions of Kantorovich type we are going to prove that the sequences $\left\{t_{n, \mu}\right\}$ and $\left\{z_{n, \mu}\right\}$, are well defined, converge to $t^{*}$ and to a solution $z^{*}$ of (4.1) respectively, and

$$
\begin{gathered}
\left|z_{n+1, \mu}-z_{n, \mu}\right| \leq t_{n+1, \mu}-t_{n, \mu}, n \geq 0 \\
\left|z^{*}-z_{n, \mu}\right| \leq t^{*}-t_{n, \mu}, n \geq 0
\end{gathered}
$$

That is, for each $\mu \geq a+\frac{2}{k},\left\{t_{n, \mu}\right\}$ is a majorizing sequence of $\left\{z_{n, \mu}\right\}[5],[3]$.

First, we give a general result on convergence of scalar sequences $\left\{t_{n, \mu}\right\}$ from Theorem 3.1 .

Lemma 4.1. Let $p(t)$ be the polynomial defined in (4.3) with two positive roots $t^{*}<t^{* *}$. Then, the sequences $\left\{t_{n, \mu}\right\}$ given by (4.4) converge to $t^{*}$. Moreover these sequences are increasing to $t^{*}$.

Proof. As in the Theorem 3.1, under the previous assumptions for $p(t)$, we have that $P_{\mu}^{\prime}(t) \geq 0$ in $\left[t_{0, \mu}, t^{*}\right]$. Therefore, $t_{n, \mu} \leq t^{*}$ for $n \geq 0$. Then, as

$$
t_{n+1, \mu}-t_{n, \mu}=-\frac{p\left(t_{n-1, \mu}\right) \mu}{p^{\prime}\left(t_{n-1, \mu}\right)\left(\mu-p\left(t_{n-1, \mu}\right)\right)} \geq 0,
$$

it follows that $\left\{t_{n, \mu}\right\}$ are increasing sequences and therefore, converge. On the other hand, as $t^{*}$ is the unique root of $p(t)=0$ in $\left[0, t^{*}\right]$, we derive that $\lim _{n \rightarrow \infty} t_{n, \mu}=t^{*}$. 
Furthermore, the convergence of the sequences $\left\{t_{n, \mu}\right\}$ has, at least, quadratic convergence. Notice that if $t^{*}=t^{* *}$ the convergence result holds, but the sequences $\left\{t_{n, \mu}\right\}$ have linear convergence.

Now, we center the study in obtaining error expressions for the sequences (4.4). So, following Ostrowski [4], derive the following error bounds.

Lemma 4.2. Let $p(t)$ be polynomial defined in (4.3). We assume that $p(t)$ has two positive roots $t^{*} \leq t^{* *}$. Let $\left\{t_{n, \mu}\right\}$ be the sequence defined by (4.4) [i] If $t^{*}<t^{* *}$, denote $\theta=\frac{t^{*}}{t^{* *}}<1$ and $d=t^{* *}-t^{*}$. Then we have

$$
\frac{d \theta^{2^{n}} H(0)^{2^{n}-1}}{1-\theta^{2^{n}} H(0)^{2^{n}-1}} \leq t^{*}-t_{n} \leq \frac{d \theta^{2^{n}} H\left(t^{*}\right)^{2^{n}-1}}{1-\theta^{2^{n}} H\left(t^{*}\right)^{2^{n}-1}},
$$

where

$$
H(t)=\frac{b \mu-\left(t^{* *}-t\right)(1-k b t)}{b \mu-\left(t^{*}-t\right)(1-k b t)}
$$

[ii] If $t^{*}=t^{* *}$, we have

$$
a_{0, \mu} \hat{H}(0)^{2^{n-1}} \leq t^{*}-t_{n} \leq \frac{a_{0, \mu}}{2^{2^{n}}}
$$

where

$$
\hat{H}(t)=1-\frac{k\left(t^{*}-t\right)^{2}}{2 \mu-k\left(t^{*}-t\right)^{2}}
$$

Proof. Let us write $a_{n, \mu}=t^{*}-t_{n, \mu}, b_{n, \mu}=t^{* *}-t_{n, \mu}, n \geq 0$. Thus

$$
p\left(t_{n, \mu}\right)=\frac{k}{2} a_{n, \mu} b_{n, \mu}, \quad p^{\prime}\left(t_{n, \mu}\right)=-\frac{k}{2}\left(a_{n, \mu}+b_{n, \mu}\right) .
$$

Then, by (4.4),

$$
a_{n+1, \mu}=t^{*}-t_{n+1, \mu}=\frac{a_{n, \mu}^{2}}{a_{n, \mu}+b_{n, \mu}}\left[1-\frac{k b_{n, \mu}^{2}}{2 \mu-k a_{n, \mu} b_{n, \mu}}\right] .
$$

and similarly,

$$
b_{n+1, \mu}=t^{* *}-t_{n+1, \mu}=\frac{b_{n, \mu}^{2}}{a_{n, \mu}+b_{n, \mu}}\left[1-\frac{k a_{n, \mu}^{2}}{2 \mu-k a_{n, \mu} b_{n, \mu}}\right] .
$$

If $t^{*}<t^{* *}$, denote $w_{n, \mu}=\frac{a_{n, \mu}}{b_{n, \mu}}$ to obtain

$$
w_{n+1, \mu}=w_{n, \mu}^{2} \frac{2 \mu-k a_{n, \mu} b_{n, \mu}-k b_{n, \mu}^{2}}{2 \mu-k a_{n, \mu} b_{n, \mu}-k a_{n, \mu}^{2}}=w_{n, \mu}^{2} H\left(t_{n, \mu}\right)
$$

where $H$ is given by (4.5). Taking into account that $H(t)$ is increasing when $t \in\left[0, t^{*}\right]$ the first part follows. 
If $t^{*}=t^{* *}$, then $a_{n, \mu}=b_{n, \mu}$. Therefore, from (4.7), we deduce

$$
a_{n+1, \mu}=\frac{a_{n, \mu}}{2}\left(1-\frac{k a_{n, \mu}^{2}}{2 \mu-k a_{n, \mu}^{2}}\right)=\frac{a_{n, \mu}}{2} \hat{H}\left(t_{n, \mu}\right),
$$

where $\hat{H}(t)$ is given by (4.6). Then, as $\hat{H}(t)$ is increasing in $\left[0, t^{*}\right]$, by recurrence, the second part holds.

Now we center the study on the sequences $\left\{z_{n, \mu}\right\}$, for $\mu \geq a+\frac{2}{k}$, defined in the Complex plane.

From now, we denote

$$
B\left(z_{0}, r\right)=\left\{z \in \mathbb{C}|| z-z_{0} \mid<r\right\}
$$

and

$$
\overline{B\left(z_{0}, r\right)}=\left\{z \in \mathbb{C}|| z-z_{0} \mid \leq r\right\}
$$

Lemma 4.3. Let $\mu \geq a+\frac{2}{k}$ and we assume [I], [II] and [III] If $|h(z)| \leq p(t)$ when $\left|z-z_{0, \mu}\right| \leq t-t_{0, \mu}<t^{*}$

then we have

[i] $|h(z)| \leq \mu$ for $z \in \overline{B\left(z_{0, \mu}, t^{*}\right)} \cap \Omega$.

[ii] $\left|\frac{1}{\mu-h(z)}\right| \leq \frac{1}{\mu-p(t)}$ when $\left|z-z_{0, \mu}\right| \leq t-t_{0, \mu}$.

Proof. As $p(t)$ is a decreasing function in $\left[0, t^{*}\right]$, given $z \in \overline{B\left(z_{0, \mu}, t^{*}\right)} \cap \Omega$ we have that there exist $t \in\left[0, t^{*}\right]$ such that $\left|z-z_{0, \mu}\right| \leq t-t_{0, \mu}<t^{*}$ and we obtain

$$
\mu \geq p(0)+\frac{2}{k} \geq p(t)+\frac{2}{k} \geq|h(z)| .
$$

Then the first part holds.

To prove [ii] notice that, as $|h(z)| \leq \mu$ for $z \in \overline{B\left(z_{0, \mu}, t^{*}\right)} \cap \Omega$, we have

$$
|\mu-h(z)| \geq|\mu-| h(z)||=\mu-|h(z)| \geq \mu-p(t),
$$

when $\left|z-z_{0, \mu}\right| \leq t \leq t-t_{0, \mu}$

Theorem 4.4. Let us assume that $k \geq\left|h^{\prime \prime}(z)\right|$ for $z \in \Omega$ and the conditions [I], [II] and [IIII] hold. Then,

[i] The sequences $\left\{z_{n, \mu}\right\}$ given by (4.2) are well defined for $\mu \geq a+2 / k$ and $n \geq 0$. Besides, they converge to $z^{*}$, solution of (4.1) in $\overline{B\left(z_{0, \mu}, t^{*}\right)} \cap \Omega$ and

$$
\begin{aligned}
\left|z_{n+1, \mu}-z_{n, \mu}\right| & \geq t_{n+1, \mu}-t_{n, \mu}<t^{*} \\
\left|z^{*}-z_{n, \mu}\right| & \leq t^{*}-t_{n, \mu}
\end{aligned}
$$

[ii] The solution $z^{*}$ is unique in $\overline{B\left(z_{0, \mu}, \frac{1}{k b}\right)} \cap \Omega$ if $t^{* *}<t^{*}$ or in $\overline{B\left(z_{0, \mu}, t^{*}\right)} \cap \Omega$ if $t^{* *}=t^{*}$. 
[iii] Besides, we have the following error bounds If $t^{*}<t^{* *}$,

$$
\left|z^{*}-z_{n}\right| \leq \frac{d \theta^{2^{n}} H\left(t^{*}\right)^{2^{n}-1}}{1-\theta^{2^{n}} H\left(t^{*}\right)^{2^{n}-1}}
$$

where $H(t)$ is given by (4.5).

If $t^{*}=t^{* *}$, we have

$$
\left|z^{*}-z_{n}\right| \leq \frac{a_{0, \mu}}{2^{2^{n}}}
$$

Proof. For $n \geq 0$, using induction, we prove

$\left[i_{n}\right]\left|\frac{1}{h^{\prime}\left(z_{n, \mu}\right)}\right| \leq-\frac{1}{p^{\prime}\left(t_{n, \mu}\right)}$,

$\left[i i_{n}\right]\left|z_{n+1, \mu}-z_{n, \mu}\right| \leq t_{n+1, \mu}-t_{n, \mu}$.

Notice that in our conditions $\left[i_{0}\right]$ holds. Furthermore, from previous Lemma and [I], [II] we derive $\left[i i_{0}\right]$.

To prove $\left[i_{n}\right]$, using $\left[i_{n-1}\right]$ and $\left[i i_{n-1}\right]$ we have

$$
\begin{aligned}
& \left|h^{\prime}\left(z_{n, \mu}\right)-h^{\prime}\left(z_{n-1, \mu}\right)\right|=\left|\int_{z_{n-1, \mu}}^{z_{n, \mu}} h^{\prime \prime}(z) d z\right| \\
\leq & \int_{t_{n-1, \mu}}^{t_{n, \mu}} p^{\prime \prime}(t) d t=p^{\prime}\left(t_{n, \mu}\right)-p^{\prime}\left(t_{n-1, \mu}\right),
\end{aligned}
$$

and therefore

$$
\begin{aligned}
& \left|1-\frac{h^{\prime}\left(z_{n, \mu}\right)}{h^{\prime}\left(z_{n-1, \mu}\right)}\right|=\left|\frac{1}{h^{\prime}\left(z_{n-1, \mu}\right)}\right|\left|h^{\prime}\left(z_{n-1, \mu}\right)-h^{\prime}\left(z_{n, \mu}\right)\right| \\
\leq & \frac{-1}{p^{\prime}\left(t_{n-1, \mu}\right)}\left[p^{\prime}\left(t_{n, \mu}\right)-p^{\prime}\left(t_{n-1, \mu}\right)\right]=1-\frac{p^{\prime}\left(t_{n . \mu}\right)}{p^{\prime}\left(t_{n-1, \mu}\right)}<1 .
\end{aligned}
$$

Then,

$$
\left|\frac{h^{\prime}\left(z_{n, \mu}\right)}{h^{\prime}\left(z_{n-1 \mu}\right)}\right|=\left|1-\left(1-\frac{h^{\prime}\left(z_{n, \mu}\right)}{h^{\prime}\left(z_{n-1, \mu}\right)}\right)\right| \geq \frac{p^{\prime}\left(t_{n, \mu}\right)}{p^{\prime}\left(t_{n-1, \mu}\right)} .
$$

So, we have

$$
\left|\frac{1}{h^{\prime}\left(z_{n, \mu}\right)}\right|=\left|\frac{1}{h^{\prime}\left(z_{n-1, \mu}\right)}\right|\left|\frac{h^{\prime}\left(z_{n-1, \mu}\right)}{h^{\prime}\left(z_{n, \mu}\right)}\right| \leq-\frac{1}{p^{\prime}\left(t_{n-1, \mu}\right)} .
$$

To prove $\left[i i_{n}\right]$ from Lemma 4.3 and $\left[i_{n}\right]$ we derive

$$
\left|z_{n+1, \mu}-z_{n, \mu}\right| \leq \frac{\left|h\left(z_{n, \mu}\right)\right|}{\left|h^{\prime}\left(z_{n, \mu}\right)\right|}\left|\frac{\mu}{\mu-h\left(z_{n, \mu}\right)}\right| \leq-\frac{p\left(t_{n, \mu}\right)}{p^{\prime}\left(t_{n, \mu}\right)} \frac{\mu}{\mu-p\left(t_{n, \mu}\right)}=t_{n+1, \mu}-t_{n, \mu}
$$

then, $\left[i i_{n}\right]$ happens and $\left\{t_{n, \mu}\right\}$ majorizes $\left\{z_{n, \mu}\right\}$ (see[3]). The convergence of $\left\{t_{n, \mu}\right\}$ (Lemma 4.1) implies the convergence of $\left\{z_{n, \mu}\right\}$ to a limit $z^{*}$. When $n \rightarrow \infty$ in (4.2), we derive $h\left(z^{*}\right)=0$.

Finally, for $p \geq 0$,

$$
\left|z_{n+p, \mu}-z_{n, \mu}\right| \leq t_{n+p, \mu}-t_{n, \mu}
$$


and taking limits when $p \rightarrow \infty$, we obtain that

$$
\left|z^{*}-z_{n, \mu}\right| \leq t^{*}-t_{n, \mu}
$$

To prove the uniqueness, notice that, $t^{*}$ is the unique fixed point of $P_{\mu}(t)$ in $\left[0, t^{\prime}\right]$ and $P_{\mu}\left(t^{\prime}\right) \leq t^{\prime},\left(t^{*} \leq t^{\prime}<1 / k b\right)$. The equality holds if and only if $t^{\prime}=t^{*}$. The result now, follows from the well known classical theorem on the existence and uniqueness of solutions of equation (4.1) via majorizing sequences given by Kantorovich [3].

Finally, [iii] follows from Lemma 4.2 .

\section{References}

[1] M. A. Hernández, and M. A. Salanova, "A family of Newton type iterative processes," Intern. J. Computer. Math., 51(1994), 205-214.

[2] A. S. Householder, The Numerical Treatment of a Single Nonlinear Equation, Mc Graw-Hill, 1970.

[3] L. V. Kantorovich and G. P. Akilov, Functional Analysis, Pergamon Press, 1982.

[4] A. M. Ostrowski, Solution of Equations in Euclidean and Banach Space, Academic Press, 1973.

[5] W. C. Rheinboldt, " A Unifed convergence theory for a Class of Iterative Process," SIAM J. Numer. Anal., 5(1968), 42-63.

Universidad de La Rioja, Dpto. de Matemáticas y Computación C/Luis de Ulloa s/n, 26004, Logroño, Spain. 